\title{
Autonomous Unmanned Surface Vehicle for Water Surface Monitoring
}

\author{
V. Nikishin, M. Durmanov \& I. Skorik \\ Sevastopol State University, Sevastopol, Russia
}

\begin{abstract}
The unmanned surface vehicle (USV) considered in this article is used for the purpose of environmental monitoring of water areas. Small size of vehicle allows to move sensors to areas close to shore line, under the low bridges where the manned boats difficult or danger to use because of damage. With its help, measurements of the required parameters are carried out directly in the studied area, or water samples are taken for further study in the laboratory. For this, it is necessary that the USV automatically move to predetermined points of the route. This article describes one of the stages in the development of an automatic control system for a given USV course. The problem of finding the steering angle for turning the USV to the required points of the route is being solved. An algorithm for visualizing the depth measurements of the exploration water area in the form of a heat map described.
\end{abstract}

\section{INTRODUCTION}

The most suitable solution for automatic surveilance of water areas is using floating unmanned surface vehicles - small-sized ship.

The USV could measure water parameters in-situ, and make sampling for ongoing more detailed laboratory research.

The main goal of current development is to design complex solution including unmanned surface vehicle (USV), custom autopilot firmware and software for real-time depth visualization. In the beginning of development we have pointed next requirements for the USV:

- Easily accessible low-cost hardware parts

- Easy USV transportation

- Monohull

- Free software libraries and IDE

- Custom autopilot

- Custom base ground station
- Propulsion high reliability

- Maneuverability

- Minimal continuous USV work over 1 hour

- Custom software for collected data postprocessing

For making USV easy to transportation we have determined the longest dimension is 1 meter, which is depends of average size of car trunk. Monohull design was chosen because of easy to proceed and USV was targeted to work in calm waters as lakes and internal bays where the high waves are rare.

\section{HULL AND HARDWARE PART}

USV was made of two main materials - fiberglass and styrofoam as hull base. The hull base was CNCmachined and glued together in one part (Figure 1). 


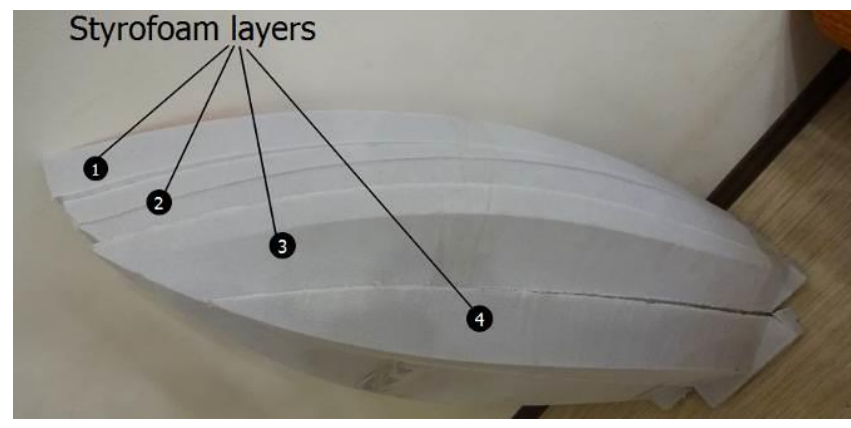

Figure 1. CNC-machined styrofoam hull base

For minimizing interaction electronic parts with water they are was placed over the top in separated covered box mounted with PVC pipes glued to the USV hull. Placing antennas as high as possible also gives advantages for reliable connection with ground station and easy access to electronic, also it worth because of magnetometer is far enough from engine containing strong magnets. Final results of the hull is shown in Figure 2.

We have selected electrical propulsion system because modern brushless and brushed motors has enough power and they are has small size suitable for using in USVs. Electrical propulsion system allows fast changing battery and it does not pollute water by any hazardous materials or exhaust gases. Our USV uses 3S (12 Volts) battery, 4074 sized brushless motor with $2000 \mathrm{kV}$, total power over $1 \mathrm{~kW}$. Motor is driven by electric speed controlled (ESC). Because of ESC is dissipates a lot of heat it is require cooling, cooling is produced by water injected by water jet.

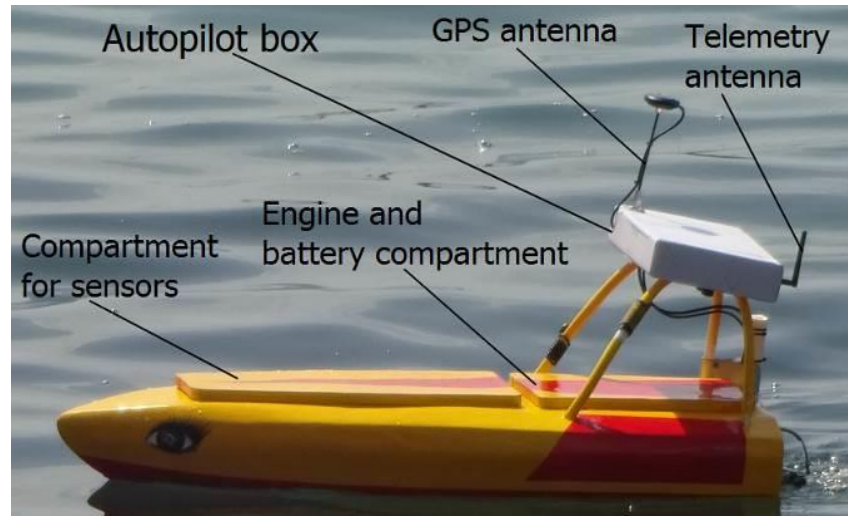

Figure 2. Unmanned surface vehicle

For steering and movement of USV we used water jet. Water jet has advantages in safety and maneuverability. Safety reason is very important if USV works near the beaches with swimming people. Because of water jet rotor located inside tube, so there are almost impossible to injure someone by rotating blades. Open propellers are more dangerous, also there are big chances that floating garbage, seaweed or fish lines could wound up on the shaft and propeller blades. Even if water jet has no safety lattice there are always problem of floating garbage and seaweed (Figure 3).

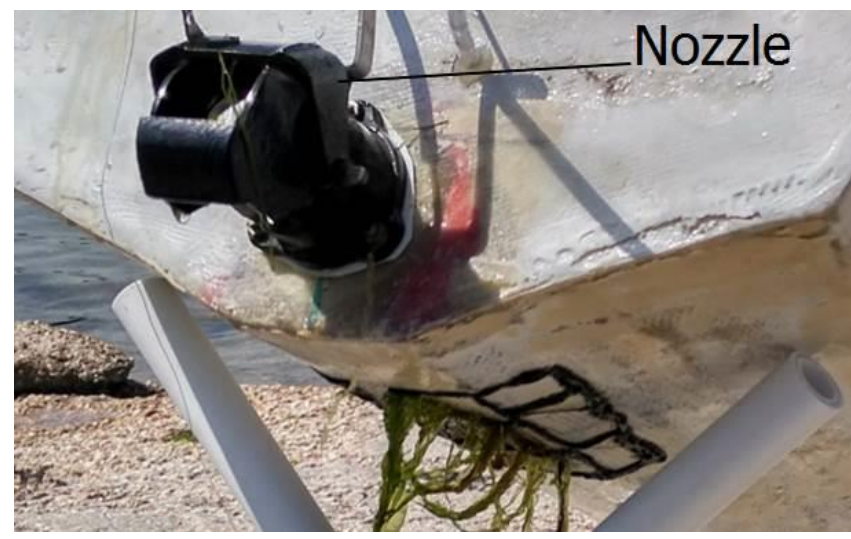

Figure 3. Seaweed in water jet

For steering of USV was used rotating nozzle. Nozzle has rotating angle near 30 degrees left and right. This allows to get very good maneuverability.

\section{AUTOPILOT}

The main purpose of autopilot is to control USV by rotating nozzle to steer it at given route point. This requires sensors (gyro, accelerometer and magnetometer) integration with microcontroller (MCU). We selected Atmega2560 on ArduinoMega board [1]. This MCU has enough interfaces for connecting with sensors and does not require high programming skill. For autonomous movement of USV main measurements is current heading and current location. Heading is measures by MEMS magnetometer connected to MCU by I2C interface. Magnetometer require calibration to minimize hard and soft iron distortion. For measurement location of USV uses GPS module U-Blox connected to MCU by UART interface. For controlling nozzle and motor ESC uses PWM signal generated by internal hardware timer [2].

The paper is overviews control system algorithm for heading hold of USV. Algorithm calculates turn angle for rudder rotation for steering the USV. This allow to steer USV in path-following mode to checkpoints stored in autopilot memory.

For correct work of USV's control system we have to consider where USV have to be turn - left or right when it moves on targeted path line. Thus, a formalized description of the algorithm is needed, according to which the calculated angle of rotation will allow the USV to rotate along the smallest arc to the azimuth required at the moment.

There are also external factors that influence the movement of USV. For example, it can be a current or wind that displaces the USV from the path line. Therefore, here it is also necessary to know the angle of rotation so that the USV can correct its movement.

But in order to make a turn, it is not enough to know only the turning angle. You want to calculate the steering angle. In the described BMA, such a device is a rotating nozzle of a water jet.

$\alpha$ rud - steering angle of rudder from $-20^{\circ}$ to $+20^{\circ}$.

In Figure 4 shows USV (1), its heading relative to the north (2) is indicated, azimuth (3), i.e. the angle to 
which the USV must turn in order to reach the path line when moving to the given checkpoint.

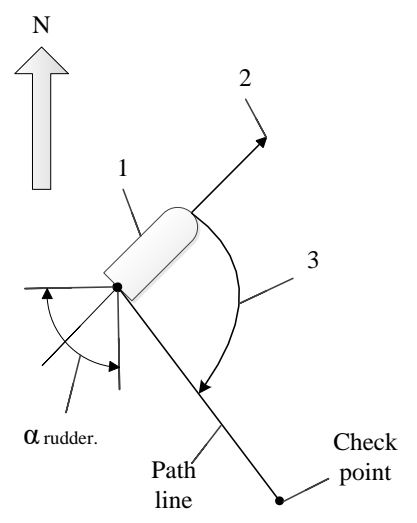

Figure 4. USV testing

Control system consist of compass and GPSreceiver. Compass in fact is 3 -axis magnetometer.

Compass data uses for heading calculation.

$$
\varphi=\operatorname{arctg}\left(\frac{Y}{X}\right) * \frac{180}{\pi}
$$

$\mathrm{X}$ and $\mathrm{Y}$ - magnetic projection of Earth magnetic field.

GPS data uses for location of USV. Azimuth to checkpoint calculates as:

$$
\begin{gathered}
\theta=\operatorname{arctg} 2\left(\sin \Delta \lambda * \cos \varphi_{2} ; \cos \varphi_{1} * \sin \varphi_{2}-\right. \\
\left.-\sin \varphi_{1} * \cos \varphi_{2} * \cos \Delta \lambda\right)
\end{gathered}
$$

where

$\Delta \lambda=\lambda_{2}-\lambda_{1}-$ longitude difference;

$\varphi_{1}$ and $\varphi 2$ - latitude difference.

First checkpoint is current USV location, second checkpoint - next checkpoint of path-line.

If the azimuth value is negative, then 360@ is added to it.

In Figure 5 shows angle rotation calculation block diagram.

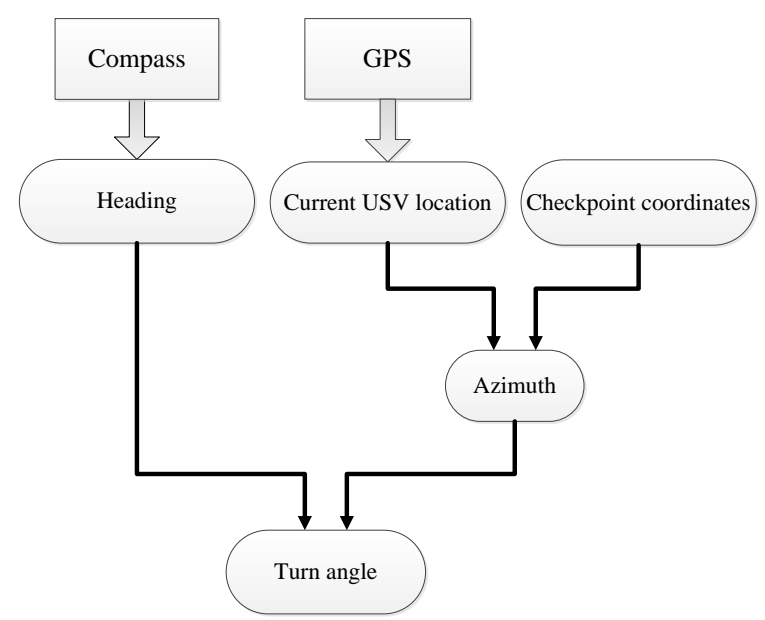

Figure 5. Angle rotation calculation block diagram
Then USV heading and azimuth uses for turn angle calculation:

$$
\Delta=\varphi-\theta
$$

Figure 6 shows the difference of azimuth and heading gives value of turn angle. But this is simplest case. Some cases where additional correction requires:

$1 \Delta$ - negative value;

$2 \Delta$ - more than $180^{\circ}$. in this case USV will turn ineffective at longest arc.

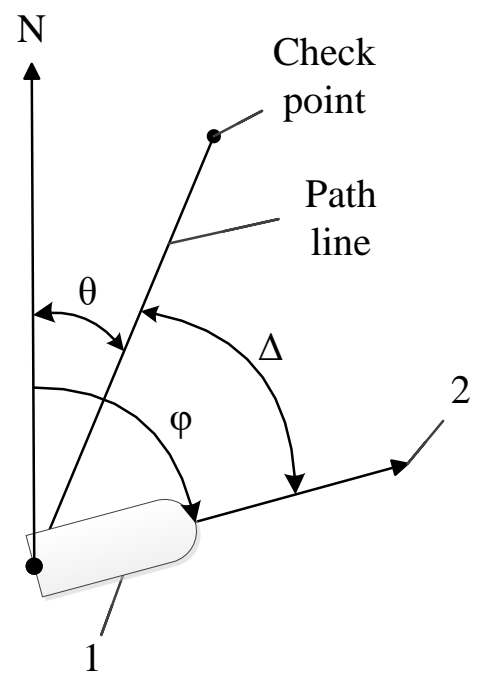

Figure 6. Navigational angles

1 - USV, 2 - current heading ๑, $\theta$ - azimuth to checkpoint, @ - turn angle, $\mathrm{N}$ - north heading

Calculation of rudder turn angle sown in Figure 7.

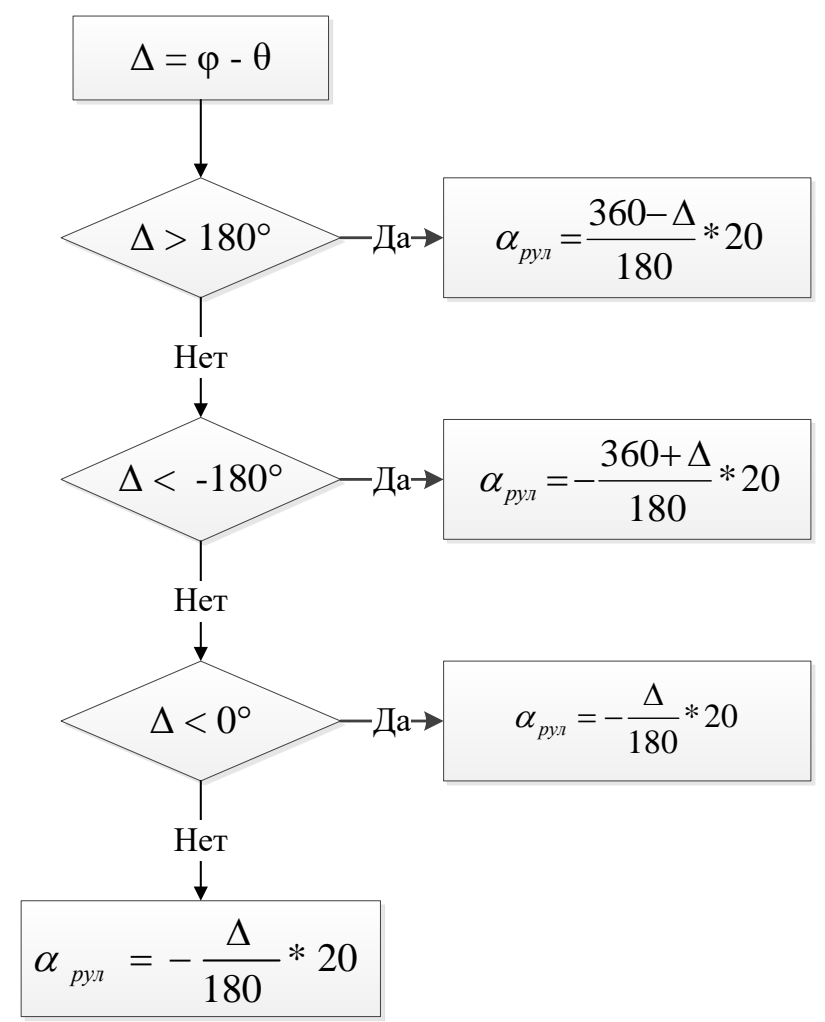

Figure 7. Rudder angle calculation block-diagram 
Given algorithm includes angle corrections. Since the rudder of USV can rotates left and right for $20^{\circ}$, angle have to be divided to $180^{\circ}$ and multiplies at $20^{\circ}$. Thus, rudder angle is calculates in allowed range from $-20^{\circ}$ to $20^{\circ}$. Negative values of rudder angle means that USV steers to the right and vice versa Figure 8

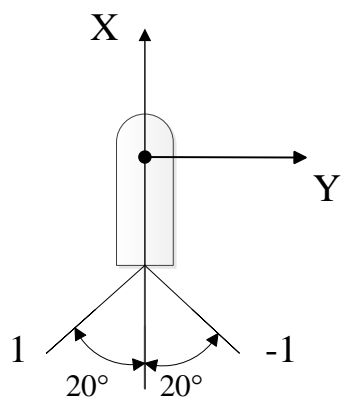

Figure 8. Rudder angle limits

The algorithm described in Figure 5 for calculating the steering angle ensures the correct movement of the USV along a path-line. During the movement, the USV will turn to the checkpoint along the smallest arc.

Also, the calculation of the steering angle allows USV to automatically correct its movement relative to the path-line.

Thus, the presented algorithm, due to its formalized form, can be integrated into the USV autopilot based on the microcontroller.

\section{SONAR}

The most useful part of USV development is integration with measurement devices. Current development is targeted to bathymetry. Selection of sonar depends on many reasons as precision, resolution, one or multi-beam ray. But in case of USV the most important is connection with MCU - sonar has to be equipped with suitable interface, in most cases this is RS-232 with NMEA standard messages [3]. We choose Garmin Echomap 42 [4], this sonar has 5 Volts NMEA interface, but it's signal is inverted. Because of Atmega2560 does not have option to invert UART signal, we have to add inverter before signal goes to MCU using NPN transistor (Figure 9).

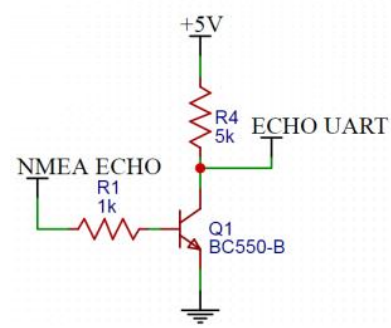

Figure 9. NMEA inverter

Placing sonar in internal compartment protects it from water sprays (Figure 10).

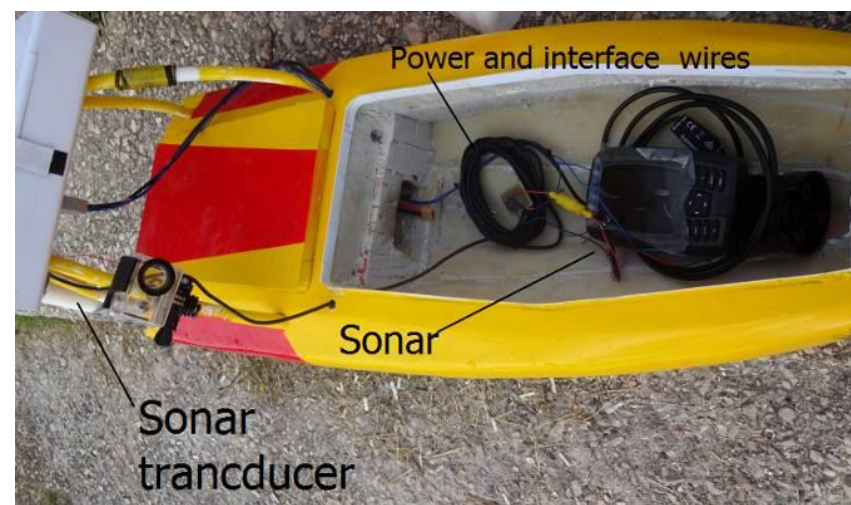

Figure 10. Placing sonar in USV

\section{POST-PROCESSING SOFTWARE DEVELOPMENT}

\subsection{Statement of the bathymetric data visualization problem}

It is proposed to visualize bathymetric data using a heat map. To do this, select a color palette and fill with the color each area with the same depth. The initial data for building a heat map are:

- geographical coordinates of points (latitude and longitude) and depth values measured by the sonar at these points;

- geographical coordinates of the boundary points of the test site, to create the boundaries of the displayed heat map;

- color palette (range and number of color shades).

To solve this problem, there are special services. For example, the Heatmaptool [5] online service allows you to use various color palettes, control the radius, zoom, and opacity of heat spots and update the map in real time. However, this service is paid and designed to visually display statistical data in the selected region.

\subsection{Heat Map algorithm}

The problem of building a heat map is to fill on the map not only the individual points at which depth measurements were made, but to fill the entire area with the color. This problem is solved using the Inverse distance weighting (IDW) [6] method, which consists in determining the weight of each point by interpolating neighboring points with a known weight at a given point.

The heat map algorithm consists from the following steps [7]:

1 Converting the geographical coordinates of the points to the $\mathrm{x}$ and $\mathrm{y}$ coordinates of the Cartesian coordinates.

2 Formation of the border of the polygon for cropping the resulting image of the heat map.

3 Implementation of the IDW method for constructing a heat map image.

4 Converting points in a Cartesian coordinates to geographical coordinates and combining a heat map with a geographical map of the area. 
The algorithm is implemented using developed software in the C \# programming language.

1 Primary processing of coordinates and bathymetric data. The heat map is built according to three parameters: geographic coordinates (longitude and latitude) and depth. USV capture this data, which is saved in a file with the .csv extension. To process bathymetric information, it is necessary to read the data from the file line by line, split them into lexemes and write the necessary parameters to the buffer. The read data are in symbolic format and for further processing and building a heat map, they are converted into real numeric format.

The dimensions of the heat map field correspond to the linear dimensions determined by the maximum and minimum values of latitude and longitude.

To process depth values, you need to convert them from text format to numeric format and convert them to a specified range.

2 Creation of polygon border for cropping the heat map image. Polygon boundary is an array of points located on the boundary of the exploration water area. The border of the polygon for cropping the image field is created at the stage of forming the dimensions of the image field and converting geographic coordinates to XOY coordinates. This is due to the fact that the polygon is also specified by geographic coordinates, which will need to be converted to XOY coordinates at the same scale.

In Figure 11 shows an image obtained using the Google Earth web application, with the help of which the geographic coordinates of this polygon were obtained.

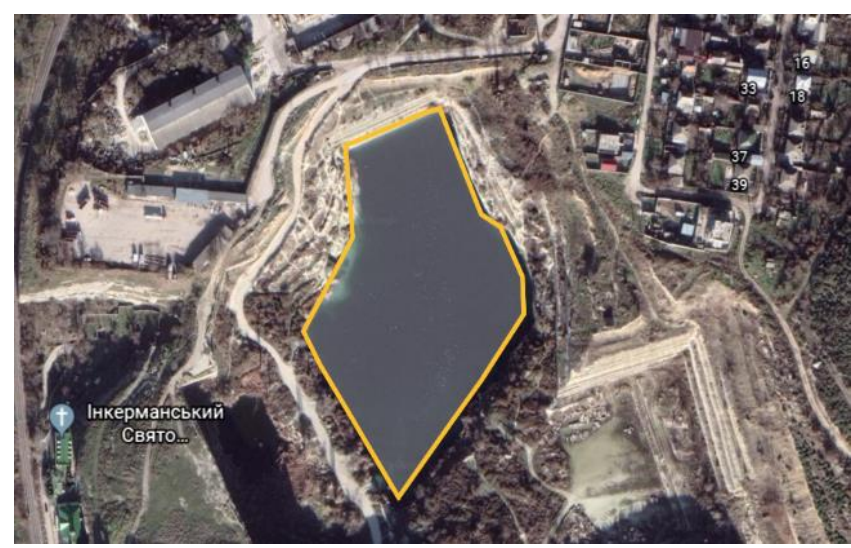

Figure 11. Exploration area of Inkerman career (Sevastopol) in Google Earth application

Considering the coordinates of the polygon corrected the linear dimensions of the plotting area of the heat map image and converted the geographic coordinates of the polygon into XOY coordinates. The image cropping contour is built using these coordinates.

3 Building a heat map using the IDW method. A heat map of the depth of an area of interest is a raster image that is obtained by calculating interpolated depth values using known depth values at fixed points. Thus, interpolation is performed in those places where we are not interested in depth, for example, on land. In this case, the resulting image must be cropped along the contour of the exploration water area. Image cropping is performed using the formed contour along the boundary points of the polygon. The color palette is chosen arbitrarily, usually warmer tones correspond to smaller depths, colder ones correspond to larger ones.

An results of building a depth map for an artificial reservoir in Inkerman (Sevastopol) is shown in Figure 12 and the coastal waters of Holland Bay (Sevastopol) is shown in Figure 13.

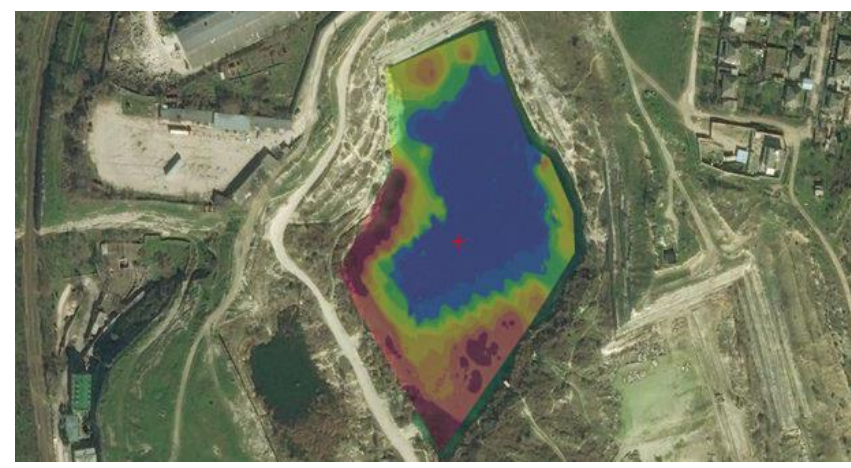

Figure 12. Heat map of Inkerman (Sevastopol)

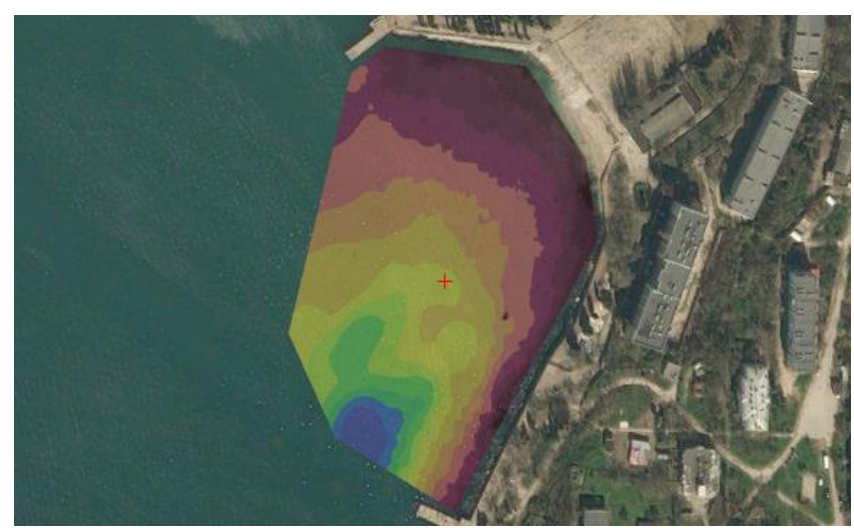

Figure 13. Heat map of Holland Bay (Sevastopol)

\section{CONCLUSION}

Developed USV shown in the paper has simple design which is can be reproduced with minimal cost. The main limitations of our USV is working only in calm waters without strong wind and waves.

Small draft of USV less than 3 inches allows to mapping depth of lakes or bays very close to shoreline, this is almost impossible with big boats with crew on board.

\section{ACKNOWLEDGEMENTS}

The reported study was funded of the internal grant by Sevastopol State University, project number 516/06-31 


\section{REFERENCES}

[1] Arduino Mega official https://store.arduino.cc/arduino-mega-2560-rev3.

[2] Nikishin V. V., Durmanov M. A. "Firmware and Software Structure Of Unmanned Naval Vehicle", Int. Conf. "Modern problems of radio electronics and telecommunications", Sevastopol State University, Sevastopol, Russia, 2019.

[3] National Marine

Electronics

Association,
[4] Garmin Echomap sonar, https://buy.garmin.com/enUS/US/p/592785.

[5] HeatmapTool.com, https://www.heatmaptool.com.

[6] GisGeography. Inverse Distance Weighting (IDW) Interpolation, https://gisgeography.com/inversedistance-weighting-idw-interpolation.

[7] Nikishin V. V., Durmanov M. A., Sizyh D. A. "Heatmap for Water Depth Visualization", Int. Conf. "Modern problems of radio electronics and telecommunications", Sevastopol State University, Sevastopol, Russia, 2019. 